\title{
Diagnostic performance of ultrasound for rotator cuff tears: a systematic review and meta-analysis
}

\author{
Weiyu Liang*, Huaiyu Wu*, Fajin Dong, Hongtian Tian, Jinfeng Xu \\ * the authors shared the first authorship
}

Department of Ultrasound, First Affiliated Hospital of Southern University of Science and Technology, Second Clinical College of Jinan University, Shenzhen Medical Ultrasound Engineering Center, Shenzhen People's Hospital, Shenzhen, China

\begin{abstract}
Aim: Rotator cuff (RC) tears are considered to be the main reason for shoulder pain. Although ultrasound is a useful method to detect it, its effectiveness when diagnosing $\mathrm{RC}$ tears has been a heated discussion. Therefore, we aimed at evaluating RC tears' ultrasound accuracy by conducting a systemically review and pooled comprehensive analysis. Materials and methods: Relevant articles up to May 2018 were searched from the Cochrane Library, Embase, and Pubmed databases. Either arthroscopy or magnetic resonance imaging (MRI) was considered as a reference standard. The results were estimated by pooled-sensitivity (P-SEN), pooled-specificity (P-SPE), pooled-diagnostic odds ratio (P-DOR), pooled-likelihood ratio+ $(\mathrm{P}-$ $\mathrm{LR}+$ ), pooled-likelihood ratio- (P-LR-), and the area under the summary receiver operating characteristic (SROC). Result: We selected seven prospective studies in accordance with the inclusion criteria that covered 554 rotator cuff tears in 868 patients. The P-SEN, P-SPE, P-LR+, P-LR-, P-DOR, area under the SROC curve of diagnostic performance of ultrasound for RC and post-test probability were $95 \%$ (95\% CI: 88 - 98), 72\% (95\% CI: $61-81), 3.41$ (95\% CI: 2.40 - 4.84), 0.08 (95\% CI: 0.03 - 0.16), 45.31 (95\% CI: 21.04 - 97.59), 89\% (95\% CI: $0.86-0.91)$, and 46\% and 2\%, respectively. Conclusion: Our metaanalysis demonstrates that ultrasound has a high efficiency for RC tears' diagnosis. It can be a promising method in patients with suspected RC tears because of its high sensitivity, specificity and diagnostic accuracy.
\end{abstract}

Keywords: ultrasound; diagnosis; meta-analysis; systematic review; rotator cuff tears.

\section{Introduction}

In the human body the shoulder can be seen as the most flexible and vulnerable joint. The primary causes of pain in shoulder and dysfunction are rotator cuff (RC)

Received 03.11.2019 Accepted 02.02.2020

Med Ultrason

2020, Vol. 22, No 2, 197-202

Corresponding author: Jinfeng Xu

Department of Ultrasound, First Affiliated Hospital of Southern University of Science and Technology, Second Clinical College of

Jinan University, Shenzhen Medical Ultrasound Engineering Center, Shenzhen Public Service Platform on Tumor Precision Medicine and Molecular Diagnosis

E-mail: xujinfeng@yahoo.com avulsions $[1,2]$. More than $72 \%$ of all shoulder pain can be attributed to RC pathology $[3,4]$. Due to the difficulty of confirming the cause of pain in the shoulder by clinical examination [5], medical imaging tends to be used to verify the diagnosis $[1,6]$. It is hard to achieve differential shoulder pathologies' diagnosis because many impairments, such as instability and shoulder impingement, could have similar symptoms $[7,8]$. Therefore, an accurate differential diagnosis is important for clinical decision making [9]. Particularly, the accurate RC tears' size and location measurement is important for the efficiency of pre-operative planning [10].

Various clinical examinations, such as arthrography, magnetic resonance imaging (MRI) and ultrasound, have been used to evaluate RC [11]. Both arthrography and MRI are the reference standards for diagnosing RC tears 
[12]. However, arthroscopy is an invasive method of inspection that is not widely accepted and MRI is timeconsuming and expensive [1,9]. Many studies have indicated that ultrasound is a useful imaging method to detect RC tears [13-18]. Compared to arthroscopy and MRI, ultrasound is relatively cheap, noninvasive, and permits a dynamic assessment [19].

Examination of the RC structure by ultrasound was first reported in the late 1970's [20]. With the emergence of high-frequency probes, the application of ultrasonography in the examination of human fine structure has become more extensive. There are many published studies on the use of ultrasound to diagnose $\mathrm{RC}$ tears, which have been divided into either complete or partial tears, and the diagnostic efficiency of ultrasound may be different in each [21]. There has been one systematic review assessing the sensitivity of ultrasound in detecting shoulder injuries [9]: however, this study separately evaluated these two types of tears. At present, no research has been undertaken on both complete and partial RC tears. Therefore, the purpose of this meta-analysis was to evaluate both types of tears in order to decide the diagnostic potential of ultrasound for RC tears.

\section{Materials and methods}

\section{Search strategy}

All relevant studies from the Cochrane Library, Embase, and Pubmed databases were retrieved. Literature was retrieved using $\mathrm{MeSH}$ and free words and English publications limited it. The search strategies applied by the Cochrane Library, Embase, and Pubmed are as follows: (infraspinatus AND tear) or (supraspinatus AND tear) or (tears AND minor AND tear) or (subscapularis AND tear) or (shoulder AND joint AND abnormalities) and ultrasound.

\section{Study selection}

We included all studies that used ultrasound to evaluate rotator cuff tears, had arthroscopy or MRI as the diagnostic criterion and directly or indirectly provided primordial data that could be used to calculate negative likelihood ratios (LR-), positive likelihood ratio (LR+), specificity and sensitivity. Cadaveric and animal trials, single case reports, incomplete research, meta-analyses and systematic reviews, studies using a population of fewer than 20 patients and research which did not have a comparison of reference standard were excluded. Two authors with the same level of expertise and experience selected literature meeting the inclusion criteria after reading the full text. A third author reviewed the search results and the assessment process and solved any discordances.

\section{Data extraction and quality assessment}

After collecting all qualified studies, we extracted the following content: reference standard, number of rotator cuff tears for analysis, number of patients, study design, year of publication, the surname of the first author, TP, FN, FP, TN, sensitivity and specificity.

The QUADAS-2 (Quality Assessment of Diagnostic Accuracy Studies-2) tool [22] was used to evaluate the quality of the eligible studies. It was made up of four parts: patient selection, index test, reference standard, and flow and timing. Every part was evaluated for bias risk and assigned a score of unclear, high, low. The reference standard domains, patient selection, index test were measured for the presence of attention on the applicability, and were likewise given a score of low, high, or unclear. If a research is evaluated by "low" in all parts regarding applicability or bias, it is good to comprehensively judge the "low risk of bias" or "low concern regarding applicability" for the research. If a research study is evaluated by "high" or "unclear" in one or more parts, it can be judged "at risk of bias" or "concerns regarding applicability".

\section{Statistical analysis}

We extracted the data from the included literature and recorded it using a four-fold table. We analyzed the data using RevMan 5.3 and Stata 12.0. RevMan 5.3 was applied in order to measure the eligible studies' methodological quality. It used stata to draw statistical graphs and pool statistical indexes such as forest plots for sensitivity, specificity, diagnostic odds ratio (DOR) with corresponding $95 \%$ confidence intervals $(\mathrm{CI})$, and the area under summary receiver operating characteristic curve (SROC). Heterogeneity of the included references was verified after statistical analysis. We used the inconsistency index $\left(\mathrm{I}^{2}\right)$ to estimate the heterogeneity across the included studies. If the $\mathrm{I}^{2}$ was $<0.5$, in the included studies, no heterogeneity was found. On the contrary, it indicated the heterogeneity's result. If heterogeneity could not be omitted, we applied a random effects model, which evaluates the combined effect's influence containing all information (sensitivity, specificity, accuracy, positive predictive value, negative predictive value, etc) [23]. The place beneath the ROC's curve shows the diagnostic test's accuracy, along with the lower accurate degree defined as an area of $0.5-0.7$, certain accuracy as an area of $0.7-0.9$, and higher accuracy with a place $>0.9$ [24].

\section{Publication bias}

The Deeks' funnel plot asymmetry test was used to evaluate potential publication bias. It was applied by regressing diagnostic log odds ratio (lnDOR) versus inverse of the square root $(1 / \mathrm{sqrt})$ of the effective sample size $\left(1 / \mathrm{ESS}^{1 / 2}\right)$ and measured through effective sample 
size. A p-value $<0.10$ for slope coefficients demonstrated the key asymmetry.

\section{Results}

Figure 1 shows the study selection process's flow chart. On the basis of the previously determined strategy of search, we searched an overall of 2,356 articles. Among these, 2,040 were from Pubmed, 273 were from Embase, and 43 were from the Cochrane Library. There were 145 duplicate materials that were excluded using ENDNOTE X7. There were 2,198 studies excluded according to the title and abstract, including eight reviews systematically and/or meta-analysis and 166 case reviews or/and researches. For the remaining 13 articles, we excluded six of them after reading the full text because they could not provide four-fold data directly or indirectly. In the end, the meta-analysis included seven articles. The included studies' features are listed in Table I.

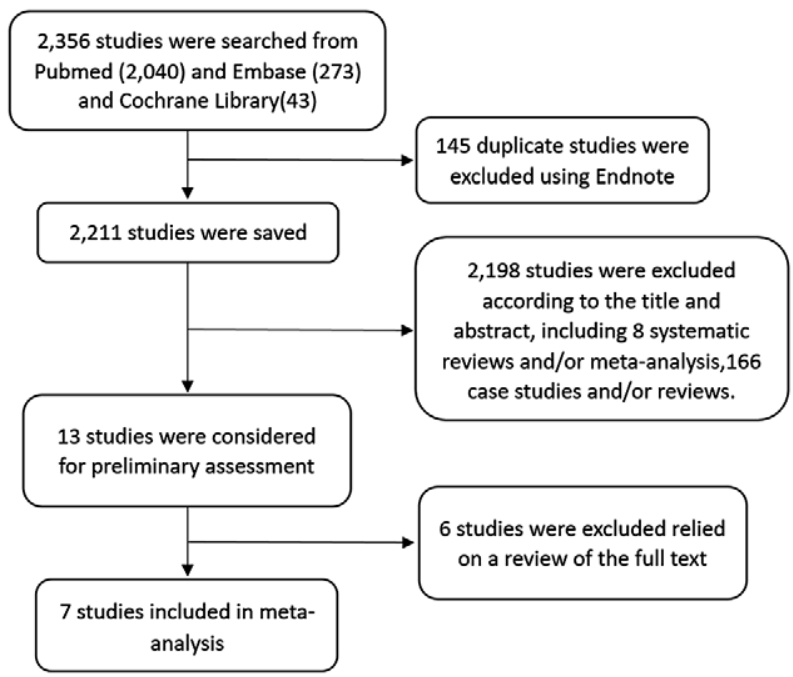

Fig 1. Flowchart of the study selection process
According to the methodological assessment from the QUADAS-2 checklist written by RevMan 5.3, patient selection, index test, reference standard, and flow and timing indicated good quality. The results are detailed in figure 2 . The selected studies' quality was relatively high and four of them had QUADAS full scores $[11,12,21,25]$.
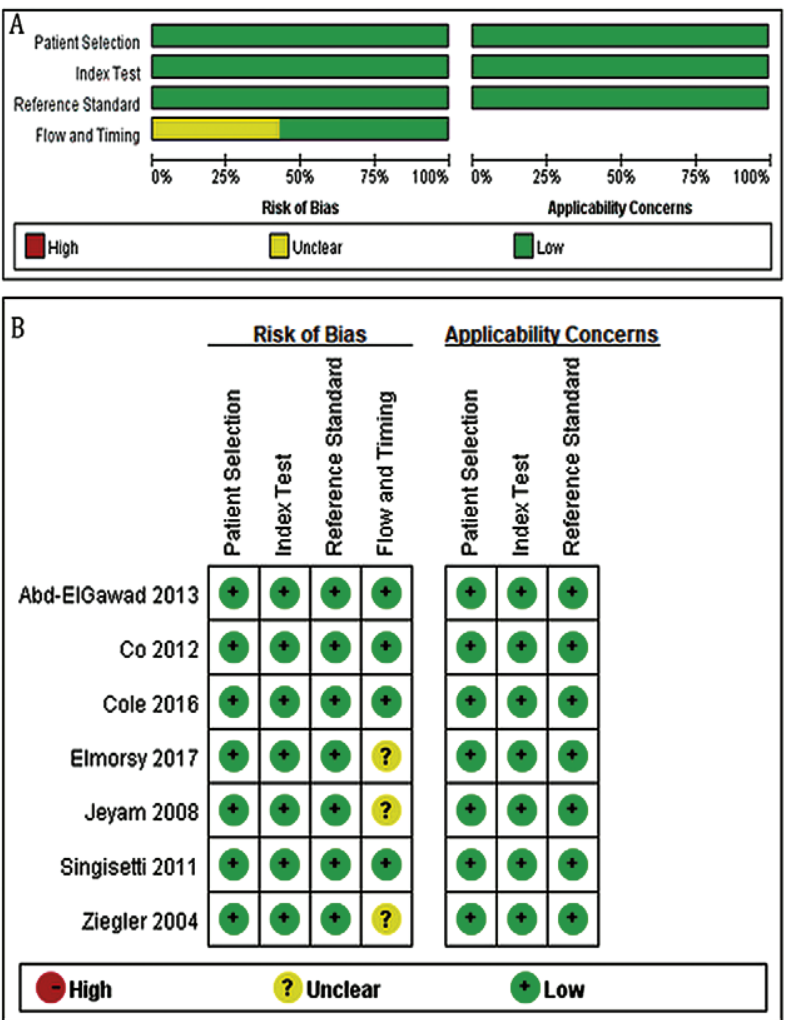

Fig 2. Quality assessment using the Quality Assessment of Diagnostic Accuracy Studies-2 (QUADAS-2) tool: A) Risk of bias and applicability concerns graph: authors' ratings for each domain are presented as percentages across included studies; B) Risk of bias and applicability concerns summary: authors' ratings for each domain for each included study.

Table I. Characteristics and diagnostic performance of included studies

\begin{tabular}{|c|c|c|c|c|c|c|c|c|c|c|}
\hline Author (year) & Country & $\begin{array}{l}\text { Number of } \\
\text { patients } \\
\text { (men) }\end{array}$ & $\begin{array}{l}\text { Full thickness } \\
\text { (partial thickness) } \\
\text { rotator cuff tears }\end{array}$ & Reference & $\mathbf{T P}$ & $\mathbf{F P}$ & $\mathbf{F N}$ & $\mathbf{T N}$ & $\begin{array}{l}\text { SE } \\
(\%)\end{array}$ & $\begin{array}{l}\text { SP } \\
(\%)\end{array}$ \\
\hline Abd-ElGawad (2013) [11] & Egypt & $40(27)$ & $23(13)$ & Arthroscopy & 35 & 2 & 1 & 2 & 97 & 50 \\
\hline Cole (2016) [12] & Australia & 238 (unclear) & $139(77)$ & Arthroscopy & 197 & 3 & 19 & 19 & 91 & 86 \\
\hline Elmorsy (2017) [19] & UK & $125(58)$ & $48(13)$ & Arthroscopy & 47 & 11 & 14 & 53 & 77 & 83 \\
\hline Co (2012) [21] & Canada & $37(22)$ & $12(10)$ & MRI & 20 & 6 & 2 & 11 & 91 & 65 \\
\hline Singisetti (2011) [25] & UK & $82(56)$ & $55(6)$ & Arthroscopy & 55 & 19 & 0 & 22 & 1 & 54 \\
\hline Jeyam (2008) [26] & UK & 64 (unclear) & $16(6)$ & Arthroscopy & 21 & 1 & 1 & 6 & 95 & 86 \\
\hline Ziegler (2004) [27] & USA & $282(173)$ & $261(1)$ & Arthroscopy & 261 & 1 & 14 & 6 & 95 & 86 \\
\hline
\end{tabular}

SE: Sensitivity, SP: Specificity, TP: True positive rate, FP: False positive rate, FN: False negative rate, TN: True negative rate 
There are three articles in which the author did not mention the time interval during the ultrasound examination and the reference standard's implication [19,26,27].

The random effects model was used to combine research data of eligible studies because the sensitivity and the LR - with 95\% CI and forest plots (fig 3a,b) displayed obvious heterogeneity, together with an I2 of $79.73 \%$ and
$73.80 \%$. Figures 3, 4a,b shows that the pooled sensitivity, specificity, LR+, LR-, DOR, area under the SROC curve of diagnostic performance of ultrasound for rotator cuff, and post-test probability were 95\% (95\% CI: $88-98)$, 72\% (95\% CI: $61-81), 3.41$ (95\% CI: $2.40-4.84), 0.08$ (95\% CI: 0.03 - 0.16), 45.31 (95\% CI: $21.04-97.59)$, $89 \%$ (95\% CI: $0.86-0.91$ ), and $46 \%$ and $2 \%$ respec-
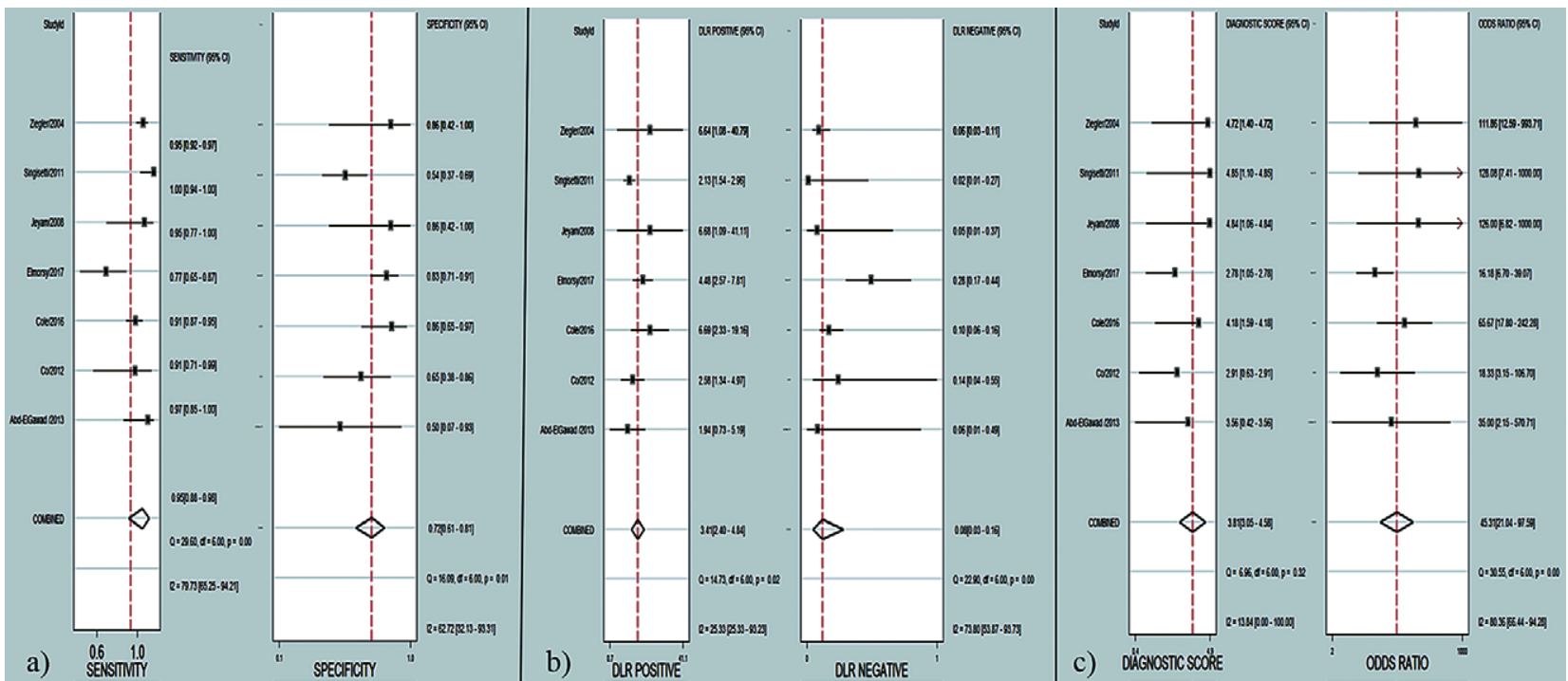

Fig 3. Forest plot for a) sensitivity and specificity of ultrasound for evaluating rotator cuff tears; b) a positive and negative likelihood ratio of ultrasound for evaluating rotator cuff tears; c) diagnostic odds ratio of ultrasound for evaluating rotator cuff tears.
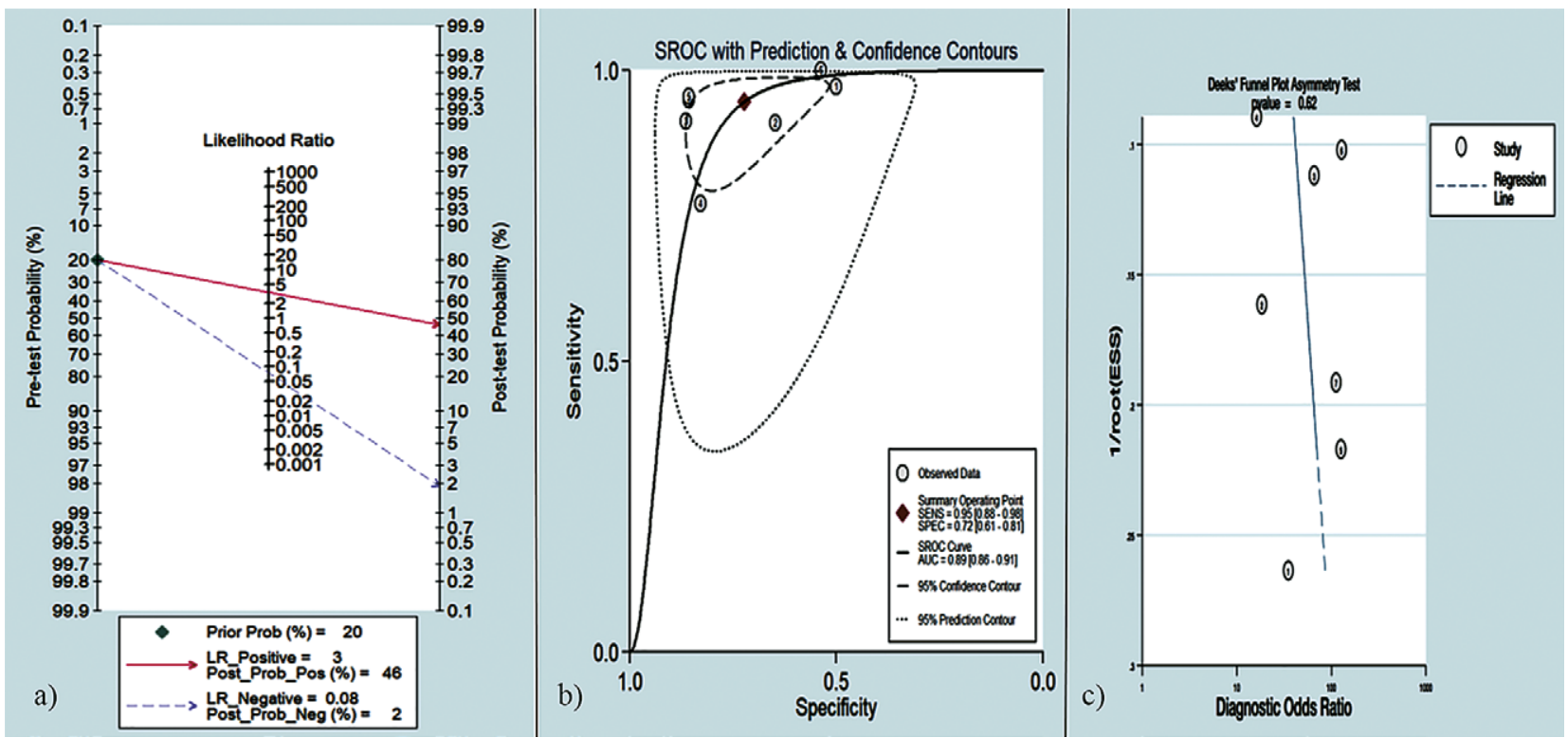

Fig 4. a) Fagan's Nomogram with pretest probability at $20 \%$; b) Hierarchical summary receiver operating characteristic curve (HSROC) with summary point, summary estimates, $95 \%$ confidence region and $95 \%$ prediction region for all included studies of ultrasound for evaluating rotator cuff tears $(n=7)$; c) Deeks' funnel plot with superimposed regression line for identifying publication bias, Log Odds Ratio versus 1/sqrt (effective sample size) (Deeks) indicates that no significant bias was found. ESS = effective sample size. 
tively. Figure 4c is a Deeks' funnel plot, which was used to indicate a systematic error in publication bias. The $\mathrm{p}$ value was 0.68 and regression line's both sides distributed the included studies, indicating that the publication bias was not apparent.

\section{Discussion}

The rotator cuff is an important structure that maintains the stability of the shoulder joint and is important in the abduction, external rotation and shoulder's internal rotation. It is the only tendonous tissue between the two bones of the shoulder joint, and is easily damaged due to its particular anatomical position and repetitive movement [28]. Many methods can be used to examine the shoulder joint. Radiography and CT are widely used in the diagnosis of bone damage and its extent, but the diagnosis of soft tissue injury is inferior to ultrasound and MRI. Although MRI is currently the most reliable, noninvasive diagnostic method for rotator cuff tears evaluation, it is an expensive imaging technique, has low reproducibility and is not suitable for use in patients with metal-mounted devices [12].

In the recent years, with the development of ultrasound technology and the emergence of high-frequency probes, ultrasound was increasingly used for musculoskeletal systems pathology. Many studies have shown that there are not obvious differences between ultrasound and MRI when detecting rotator cuff tears $[19,26,29]$. However, though ultrasound is inexpensive, noninvasive and convenient, its effectiveness when diagnosing RC tears is still controversial. We found that the combined sensitivity, specificity, LR- and DOR of the included studies show higher heterogeneity $\left(\mathrm{I}^{2}>50 \%\right)$. Even so, ultrasound had a high sensitivity and credible specificity when diagnosing the rotator cuff tear. We also found a high LR+, DOR, certain accuracy and a lower LR-. The LR is a composite indicator reflecting sensitivity and specificity, which can better reflect the diagnostic value of diagnostic experiments [30].

Although we systematically evaluated the inclusion of literature using a rigorous process, there are still deficiencies. For example, Elmorsy et al did not mention the time interval between the ultrasound exam and the reference standard's implementation [19], which could generate the bias of patient flow. In addition, our study did not contain unpublished and non-English articles. Finally, the studies we included are from different regions, which is likely to produce differences in compliance. In all, compliant bias, language, and publication are shown.

This meta-analysis demonstrates that combined sensitivity and LR- are of a high degree of heterogeneity and we have three explanations for this. First, a portion of the included studies was selected for patients with clinically, highly suspected rotator cuff tears [19,21]. Second, some papers included in our study individually analyzed partial and complete tears [25-27], but we combined the two for analysis. Third, the frequency of the probe used and the diagnostic level of the operating physician was different in each study. These factors are likely to introduce bias and represent limitations to this study.

\section{Conclusion}

In conclusion, our meta-analysis demonstrates that ultrasound has a high efficiency of diagnosis for rotator cuff tears. As for patients, it can be a promising approach with suspected rotator cuff tears due to its high diagnostic accuracy and sensitivity specificity.

\section{Conflicts of interest: none}

Acknowledgements: The project was supported by the National Natural Science Foundation of China (NO.81771841) and the Commission of Scientific and Technology of Shenzhen (No. JCYJ20170307095706 970).

\section{References}

1. Milosavljevic J, Elvin A, Rahme H. Ultrasonography of the rotator cuff:a comparison with arthroscopy in one-hundredand-ninety consecutive cases. Acta Radiol 2005;46:858865 .

2. Seibold CJ, Mallisee TA, Erickson SJ, Boynton MD, Raasch WG, Timins ME. Rotator cuff: evaluation with US and MR Imaging. Radiographics1999;19:685-705.

3. Macfarlane GJ, Hunt IM, Silman AJ. Predictors of chronic shoulder pain:a population based prospective study. J Rheumatol 1998;25:1612-1615.

4. Mitchell C, Adebajo A, Hay E, Carr A. Shoulder pain: diagnosis and management in primary care. BMJ 2005;331:1124-1128.

5. Naqvi GA, Jadaan M, Harrington P. Accuracy of ultrasonography and magnetic resonance imaging for detection of full thickness rotator cuff tears. Int J Shoulder Surg 2009;3:94-97.

6. Roberts CS, Walker JA 2nd, Seligson D. Diagnostic capabilities of shoulder ultrasonography in the detection of complete and partial rotator cuff tears. Am J Orthop (Belle Mead NJ) 2001;30:159-162.

7. Mack LA, Gannon MK, Kilcoyne RF, Matsen RA 3rd. Sonographic evaluation of the rotator cuff. Accuracy in patients without prior surgery. Clin Orthop Relat Res 1988;234:21-27.

8. Friedman RL, Hidalgo HJ, Gilmer PW, et al. Ultrasonography of the rotator cuff: analysis of results in a community setting. J Shoulder Elbow Surg1993;2:22-6. 
9. Smith TO, Back T, Toms AP,. Hing CB. Diagnostic accuracy of ultrasound for rotator cuff tears in adults: A systematic review and meta-analysis. J Clin Radiology 2011;66:10361048.

10. Farin PU, Kaukanen E, Jaroma H, Väätäinen U, Miettinen $\mathrm{H}$, Soimakallio S. Site and size of rotator-cuff tear. Findings at ultrasound, double-contrast arthrography, and computed tomography arthrography with surgical correlation. J Invest Radiol 1996;31:387-394.

11. Abd-ElGawad EA, Ibraheem MA, Fouly EH. Evaluation of supraspinatus muscle tears by ultrasonography and magnetic resonance imaging in comparison with surgical findings. Egypt J Radiol Nucl Med 2013;44:829-834.

12. Cole B, Twibill K, Lam P, Hackett L, Murrell GA. Not all ultrasounds are created equal: general sonography versus musculoskeletal sonography in the detection of rotator cuff tears. Shoulder Elbow 2016;8:250-257.

13. Allen GM, Wilson DJ. Ultrasound of the shoulder. Eur J Ultrasound 2001;14:3-9.

14. Dinnes J, Loveman E, McIntyre L, Waugh N. The effectiveness of diagnostic tests for the assessment of shoulder pain due to soft tissue disorders: a systematic review. Health Technol Assess 2003;7:1-166.

15. Jacobson JA, van Holsbeeck MT. Musculoskeletal ultrasonography. Orthop Clin North Am 1998;29:135-167.

16. Middleton WD, Teefey SA, Yamaguchi K. Sonography of the rotator cuff: analysis of interobserver variability. AJR Am J Roentgenol 2004;183:1465-1468.

17. Martinoli C, Bianchi S, Prato N, et al. US of the shoulder: non-rotator cuff disorders. Radiographics 2003;23:381-401.

18. de Jesus JO, Parker L, Frangos AJ, Nazarian LN. Accuracy of MRI, MR arthrography, and ultrasound in the diagnosis of rotator cuff tears: a meta-analysis. AJR Am J Roentgenol 2009;192:1701-1707.

19. Elmorsy A, Keightley A, Flannery M. Accuracy of Ultrasonography (US) and Magnetic Resonance Imaging (MRI) in Detection of Rotator Cuff Tears in District General Hospital. Pol J Radiol 2017;82: 634-637.
20. Seltzer SE, Finberg HJ, Weissman BN, Kido DK, Collier $\mathrm{BD}$. Arthrosonography: gray-scale ultrasound evaluation of the shoulder. Radiology 1979;132:467-468.

21. Co S, MD, Bhalla S, Rowan K, Aippersbach S, Bicknell S. Comparison of 2- and 3-dimensional shoulder ultrasound to magnetic resonance imaging in a community hospital for the detection of supraspinatus rotator cuff tears with improved worktime room efficiency. Can Assoc Radiol J 2012:63:170-176.

22. Whiting PF, Rutjes AW, Westwood ME, et al. QUADAS-2: A revised tool for the quality assessment of diagnostic accuracy studies. Ann Intern Med 2011;155:529-536.

23. Higgins JP, Thompson SG, Deeks JJ, Altman DG. Measuring inconsistency in meta-analyses. BMJ 2003;327:557560.

24. Swets JA. Measuring the accuracy of diagnostic systems. Science 1988;240:1285-1293.

25. Jeyam M, Funk L, Harris J. Are shoulder surgeons any good at diagnosing rotator cuff tears using ultrasound?: A comparative analysis of surgeon vs radiologist. Int J Shoulder Surg 2008;2:4-6.

26. Singisetti K, Hinsche A. Shoulder ultrasonography versus arthroscopy for the detection of rotator cuff tears: analysis of errors. J Orthop Surg (Hong Kong) 2011;19:76-79.

27. Ziegler DW. The use of in-office, orthopaedist-performed ultrasound of the shoulder to evaluate and manage rotator cuff disorders. J Shoulder Elbow Surg 2004;13:291297.

28. Moosmayer S, Heir S, Aaser P, Smith HJ. Ultrasound examination of the shoulder a method description. Tidsskr Nor Laegeforen 2004;124:177-180.

29. Modi CS, Smith CD, Ho K, et al. Accuracy of highresolution ultrasonography in the diagnosis of articular side partial thickness rotator cuff tears. Shoulder Elbow 2010;2:267-270.

30. Bürkner PC, Doebler P. Testing for publication bias in diagnostic meta-analysis: a simulation study. Stat Med 2014;18:3061-3077. 